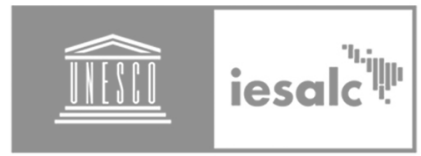

\title{
Unesco
}

Instituto Internacional

para la Educación Superior

en América Latina

y el Caribe
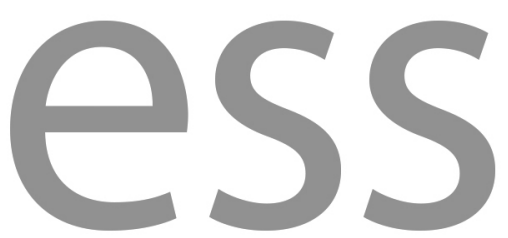

?

Educación

Superior y

Sociedad

Vol. 33 No. 2 (2021)

-

$\bullet$

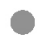

$\bullet$
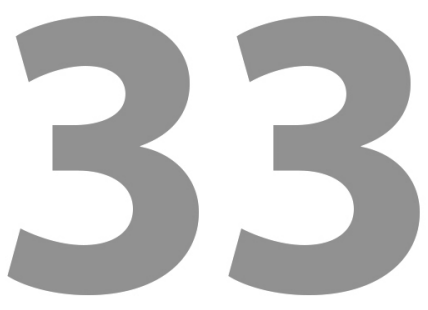

Incluye Dossier Temático:

Desafíos de la educación superior frente a la pandemia de Covid-19 en América Latina y el Caribe 


\section{Efectos en los procesos de escolarización superior: un acercamiento al escenario educativo en tiempos de distanciamiento social}

Jaqueline Guadalupe Guerrero, José Duarte Méndez,

Diana Moguel Ruz, José Canto Ramírez, Martín Breton de la Loza

\section{RESUMEN}

El año 2020 ha quedado marcado en la memoria de toda la humanidad, debido al distanciamiento social ocasionado por el virus SARS-CoV-2, las repercusiones han sido catastróficas, y el ámbito educativo no ha sido la excepción, propiciando que se afronte un reto inédito: pasar de lo presencial a lo virtual. Este estudio tiene como objetivo identificar el efecto de las estrategias implementadas en instituciones de educación superior en respuesta a este escenario inesperado de contingencia sanitaria, en el periodo de marzo 2020 a enero 2021. El método de aula invertida y las nuevas tecnologías de la información y la comunicación han tomado importancia en la docencia virtual. La metodología utilizada es un enfoque mixto con alcance descriptivo y diseño no experimental y transversal. Se realizó un análisis de las estrategias implementadas por cuatro instituciones (tres públicas y una privada) ubicadas en San Francisco de Campeche, Campeche, México. La muestra fue de 134 estudiantes; para la recolección de datos se aplicaron dos instrumentos: ficha de identificación de creación propia y test de estrés laboral adaptado del cuestionario de problemas psicosomáticos. Se identificó adaptación a la nueva normalidad, autopercepción de ansiedad, desempeño académico y consolidación del aprendizaje.

Palabras clave: Pandemia; Aula invertida; Distanciamiento social; Educación Superior.

\section{Effects on higher education processes: an approximation to the educational scenario in times of social distancing}

\section{ABSTRACT}

The year 2020 marked the memory of all humanity due to the social distancing caused by the SARS-CoV-2 virus, the repercussions have been catastrophicand 
the educational field has been no exception, leading to an unprecedented challenge: $g$ o from face-to-face to virtual modalities. This study aims to identify the effect of the strategies implemented in higher education institutions, in response to this unexpected scenario of health contingency from March 2020 to January 2021. The flipped classroom method and the new information and communication technologies have become important in virtual teaching. The methodology used is a mixed approach with a descriptive scope, and a non-experimental cross-sectional design. An analysis was performed on the strategies implemented by four institutions (three public and one private) located in San Francisco de Campeche, Campeche, Mexico. The sample was based on 134 students; and two instruments were applied to collect data: the identification card of own creation and the work stress test, adapted from the questionnaire on psychosomatic problems. Adaptation to the new normal, self-perception of anxiety, academic performance and consolidation of learning were identified.

Keywords: Pandemic; Flipped classroom; Social distancing; Higher Education.

\section{Efeitos sobre os processos de ensino superior: uma aproximação ao cenário educacional em tempos de distanciamento social}

\section{RESUMO}

O ano de 2020 ficou marcado na memória de toda a humanidade por conta do distanciamento social causado pelo vírus SARS-CoV-2, as repercussões foram catastróficas e o campo educacional não foi a exceção, trazendo à tona um desafio inédito: pasar do modelo presencial para o virtual. Este estudo tem como objetivo identificar o efeito das estratégias implementadas nas instituições de ensino superior, em resposta a este cenário inesperado de contingência sanitária, no período de março de 2020 a janeiro de 2021. O método da aula invertida e as novas tecnologias de informação e comunicação tornaram-se importantes no ensino virtual. A metodologia utilizada é uma abordagem mista, de escopo descritivo, não experimental e de delineamento transversal. Uma análise foi feita sobre as estratégias implementadas por quatro instituições (três públicas e uma privada) localizadas em San Francisco de Campeche, Campeche, México. A amostra foi de 134 estudantes; dois instrumentos foram aplicados para a coleta de dados: carteira de identidade própria e teste de nível de estresse ocupacional adaptado do questionário sobre problemas psicossomáticos. 
Adaptação ao novo normal, autopercepção da ansiedade, desempenho acadêmico e consolidação da aprendizagem foram identificados.

Palavras-chave: Pandemia; Aula invertida; Distanciamento social; Educação Superior.

\section{Effets des processus d'enseignement supérieur: un rapprochement au scénario éducatif en période de distanciation sociale.}

\section{RESUMÉ}

L'année 2020 a marquée la mémoire de toute l'humanité,en raison de la distanciation sociale causée par le virus SARS-CoV-2, les répercussions ont été catastrophiques et le domaine éducatif n'a pas été exception, provoquant un défi sans précédent: passer du modèle présentiel au virtuel Cette étude vise à identifier l'effet des stratégies mises en œuvre dans les établissements d'enseignement supérieur en réponse à ce scénario inattendu de contingence sanitaire, dans la période de mars 2020 à janvier 2021. La méthode de la classe inversée et les nouvelles technologies de l'information et de la communication sont devenues importantes dans l'enseignement virtuel. La méthodologie utilisée est une approche mixte de portée descriptive et de conception non expérimentale et transversale. Une analyse a été faite sur les stratégies mises en œuvre au sein de quatre institutions (trois publiques et une privée) situées à San Francisco de Campeche, Campeche, Mexique. L'échantillon était de 134 étudiants et deux instruments ont été utilisés pour collecter les données: la carte d'identité auto-créée et le test de stress au travail adapté du questionnaire des problèmes psychosomatiques. L'adaptation à la nouvelle normalité, la perception de l'anxiété par soi-même, les performances scolaires et la consolidation des apprentissages se sont des éléments qui ont été identifiés.

Mots-clés: Pandémie; Classe Inversée; Distanciation Sociale; Enseignement Supérieur. 


\section{INTRODUCCIÓN}

El año 2020 ha dejado una huella muy profunda en la vida de las personas en el mundo, invadidos por el miedo al contagio del virus SARS-CoV-2, el cual surgió a finales del año 2019 en Wuhan, China. Debido a la ubicación geográfica de este país, parecía muy lejana la posibilidad de que llegara a México, lo cual motivó que el año 2020 se iniciara con toda confianza y tranquilidad; paulatinamente este escenario fue cambiando, el virus iba avanzando y ampliando su cobertura en todo el mundo, surgiendo el temor al contagio y a perder la vida. En este contexto, los líderes mundiales tomaron decisiones drásticas que implicaron detener el mundo, lo impensable ya era una realidad: se ordenó confinamiento sanitario, disfrazado de voluntario "por motivos de salud", surgiendo el aislamiento social, ocasionando que el sector productivo y económico se detuvieran, las escuelas fueron cerradas y los hospitales públicos se limitaron a la atención de urgencia, transformándose en hospitales COVID, al ser insuficientes, el Gobierno Federal estableció convenios con los hospitales del sector privado, para garantizar la atención de las personas.

Este distanciamiento social derivado de la contingencia sanitaria por el COVID 19 , ha propiciado que la educación superior afronte un reto inédito, consistiendo en transitar de la modalidad presencial a la docencia en ambientes virtuales sin la preparación adecuada, no estaba previsto este escenario de confinamiento y distanciamiento social.

La tecnología ha estado presente en las instituciones de educación superior, tanto públicas como privadas, desde hace algunas décadas, aproximadamente a mediados del siglo XX, con la inclusión del proyector de acetatos, que había que solicitarlo en la Dirección. A finales del siglo XX se adquieren los primeros proyectores digitales (conocido como "cañón") con el complemento de la computadora, para proyectar las presentaciones en diapositivas del material de clase. Poco a poco se fueron instalando en cada salón de clases, para ser conectados al equipo de cómputo, en los salones que no los tienen instalados, se solicitan en la Coordinación, esto es representativo en todas las escuelas que participan en este estudio.

En la Universidad Autónoma de Campeche, aproximadamente en 2011 (siglo XXI) se adquieren las pizarras digitales, ubicándose sólo en algunos salones, debido al alto costo fue limitada la compra a uno o dos por programa educativo 
(licenciatura). Se considera un dispendio de recurso económico, porque se adquirieron y se instalaron, pero nunca se usaron, faltó la capacitación al docente en el uso de esta tecnología, hasta el momento los pizarrones continúan en los salones sin ser utilizados.

Los elementos que constituyen una institución educativa sólida son: el plan de estudios, la calidad de sus docentes y la infraestructura, en un marco de autoridades académicas verdaderamente comprometidas con la calidad en el servicio que se ofrece a los estudiantes, que finalmente son los clientes que lo reciben, es por ello que debe evitarse la simulación, debido a que es el peor de los males que se tiene en el ámbito educativo.

Indudablemente la figura que representa a la institución y da la cara al estudiante son los docentes, es evidente que son expertos en su área de conocimiento, sin embargo se observan características que disminuyen su nivel de compromiso, una de ellas es que existen profesores que tienen un trabajo adicional, ante la necesidad de elevar su ingreso tienen compromisos en dos o más instituciones, ocasionando que dispongan de poco tiempo para dedicarle a la responsabilidad académica, la cual no solo implica el proceso enseñanzaaprendizaje, sino que también se encuentran incluidas reuniones y actividades de capacitación de interés institucional. Lo cual implica una mayor dedicación de tiempo para capacitarse en temas de actualización docente y uso de nuevas tecnologías aplicadas a la educación.

También existe el tema de que algunos docentes no invierten en la adquisición de equipos personales de cómputo, creen que es obligación de la institución darles todas las herramientas necesarias para el desempeño de su labor, pudiendo ser esta la razón por la cual carecen de computadora con cámara y capacidad para soportar las aplicaciones indispensables para el desempeño de sus funciones.

Con la llegada del Coronavirus, surge el término "nueva normalidad", ante lo cual se han tomado acciones, siendo la principal llevar las clases a la modalidad virtual y desde la residencia de los docentes, esta adaptación trae cambios importantes como son: la pérdida de la privacidad del docente, transformar su casa en un salón de clases, su espacio privado ahora es público, su tiempo ha dejado de ser propio, gran parte ahora es de los estudiantes, con el uso de las aplicaciones debe estar disponible casi las 24 horas del día, los siete días de la semana para atender las dudas e inquietudes, siendo prioritario continuar con la capacitación en el tema del uso de herramientas informáticas. 
El estudiante, también ha tenido complicaciones para transitar de lo presencial a lo virtual iniciando por el escenario económico, hay que recordar que la economía se paralizó, se perdieron demasiados empleos, ante esta situación, los jóvenes salieron a la búsqueda de empleos para aportar a la economía familiar, otro aspecto es la conectividad a internet y el equipo de cómputo, el cual debe tener cámara y audio aceptable, para escuchar y participar en las sesiones educativas. El hecho de tomar las clases en casa, también ha implicado el reto de disponer de privacidad en su casa, sobre todo si se tiene en cuenta que en los hogares tienen familiares en diversos niveles educativos y que pueden coincidir en el día y hora en que toman clases y tal vez viven en casas pequeñas, por lo que pudiera ser un motivo para mantener su cámara cerrada.

Sumando a lo anterior el hecho de que el comercio informal creció exponencialmente y ahora es muy común escuchar en las calles y a toda hora del día los vendedores que a través de triciclos, motos, autos y camiones van promoviendo, a viva voz o con el uso de otras herramientas radiofónicas, sus productos y servicios como: tortillas, agua purificada, gas, entre otros productos, además del camión recolector de basura, lo cual dificulta la concentración de los estudiantes y docentes durante la sesión, siendo el ruido un agente estresor que pudiera repercutir en la alteración del estado socioemocional al no poder concentrarse, sobre todo cuando están exponiendo o realizando alguna aportación al tema de análisis; tal vez esta sea una de las razones por las que los estudiantes mantienen cerrado su micrófono.

Por su parte, los rectores y directores de las universidades, en su calidad de autoridad académica, en la medida de sus posibilidades presupuestales, han realizado acciones de capacitación a su personal, para dotarlos de estrategia que les permita mejorar su desempeño profesional en el uso de las herramientas de Google Suite.

Este artículo se realiza con el objetivo identificar el efecto de las estrategias implementadas en instituciones de educación superior, en respuesta a este escenario inesperado de contingencia sanitaria, en el periodo de marzo 2020 a enero 2021. En este sentido surge la siguiente pregunta de investigación: ¿cuál es la estrategia implementada por la autoridad educativa y los estudiantes, para afrontar el reto del distanciamiento social en tiempos de pandemia, por parte de las instituciones de educación superior de la ciudad de San Francisco de Campeche, Campeche, México, en el periodo marzo 2020 a enero 2021? 
Responder dicha pregunta, permitirá identificar las estrategias implementadas por la autoridad académica como respuesta a la contingencia sanitaria, permitiendo conocer los esfuerzos que los docentes están realizando en favor de los estudiantes quienes son la razón de ser del trinomio educativo, con la intención de lograr la identificación y análisis del efecto que está dejando la contingencia sanitaria en la educación superior.

\section{ANTECEDENTES}

Con la intención de ampliar el conocimiento sobre el tema, se ha realizado una selección de nueve investigaciones relacionadas con el estudio, procedentes de los países de: Colombia, Argentina, Perú, España y México (dos estudios), así como tres instituciones que han realizado importantes esfuerzos por tener una radiografía de lo que está sucediendo, ellos son: Asociación Internacional de Universidades (IAU por sus siglas en inglés) y la Asociación Iberoamericana de Docencia Universitaria en México (AIDU México), seguidamente se describen los aspectos relevantes:

El primer estudio seleccionado fue realizado en Colombia y presenta una recopilación de diferentes acciones políticas educativas, se titula: "COVID- 19. Acciones globales frente al cierre de escuelas durante una pandemia" de la autoría de (García, et al., 2020), se aprecian las diferentes estrategias de enseñanza, implementadas en otros países como por ejemplo en India que se utilizó Byju, Topper y UNacademy; o en El Salvador, que se empleó Virtual Classroom, Edmodo, Trello y Slack.

Un aspecto que se puede distinguir en esta revisión, es la alianza que existe no sólo entre el gobierno y las instituciones sino también con empresas particulares que tiene como objetivo crear plataformas y/o aplicaciones educativas como es el caso de la plataforma Feide en Noruega. Además, se menciona un factor importante que ya existía pero que con la pandemia se hizo más evidente: la desigualdad económica, que ha sido un obstáculo en los procesos de escolarización. Este estudio es relevante para este trabajo de investigación debido a que aporta estrategias innovadoras utilizadas por docentes y gobiernos de otros países; que podrían ser implementadas en México como alternativas a las que ya se emplean actualmente. 
La siguiente investigación fue realizada en Argentina llamada "Uso de TIC en prácticas pedagógicas de docentes de la Facultad de Psicología de una universidad pública argentina" en el cual se encuentra una encuesta realizada a 63 docentes de la cual se obtuvieron los siguientes resultados: el $86 \%$ está de acuerdo en que los alumnos tengan el correo electrónico del docente, casi el $43 \%$ nunca ha llevado un curso en la modalidad virtual, el $40 \%$ ha utilizado Facebook como medio de comunicación con los alumnos, el $49 \%$ respondió que nunca consideró utilizar WhatsApp para comunicarse con los estudiantes, y por último, el $49 \%$ nunca ha utilizado una plataforma virtual para dar su cátedra (Borgobello, et al., 2019). Con base en esta investigación, podemos afirmar que hace apenas dos años, el uso de las TIC no era considerado fundamental por los maestros al momento de enseñar sus respectivas materias; esta información debe tomarse en cuenta para poder comparar cómo es que la pandemia obligó a los docentes a prepararse y certificarse en distintas plataformas virtuales.

El estudio"Actitud de los estudiantes universitarios frente a la educación virtual en tiempos de la pandemia de COVID-19" realizado en la Universidad Nacional Amazónica de Madre de Dios, en Perú, demostró que la mayoría de estudiantes presentan una actitud de indiferencia frente a la educación virtual, así como también, evidencian una actitud de rechazo ante el soporte pedagógico y técnico y como se incrementaron sus niveles de estrés y ansiedad al desenvolverse virtualmente en comparación con la modalidad presencial (Estrada-Araoz, et al., 2020). Esta información resulta relevante debido a que muestra que la pandemia afecta psicosocialmente a los estudiantes y que su falta de interés en el aula digital disminuye su rendimiento académico.

De igual manera, se identificó otro estudio "Políticas educativas, en tiempo de Coronavirus: la confrontación ideológica en España", de la autoría de Enrique Javier Díez Gutiérrez, Katherine Gajardo Espinoza, que hace mención que: las administraciones educativas han establecido instrucciones y normativas para regular el fin del curso escolar, su evaluación y el inicio del nuevo curso, ante la situación de suspensión de clases durante el tercer trimestre y el confinamiento decretado por la pandemia del Covid-19. Estos 20 documentos y 369 páginas permiten conocer las distintas políticas educativas adoptadas por las Comunidades Autónomas en España. Se ha utilizado para ello un análisis crítico del discurso. Los resultados indican que las normativas se encuadran entre dos grandes enfoques: uno más "progresista" centrado en asentar los 
aprendizajes básicos y relevantes, el apoyo emocional y la evaluación formativa, donde la tercera evaluación no presencial se consideraba "no calificable". Otro más "conservador" que, apuesta por seguir avanzando contenidos, evaluarlos, estableciendo no promocionar ni titular con asignaturas suspensas. No obstante, hay una serie de medidas que han generado un consenso pedagógico muy positivo de cara a las futuras políticas educativas en este país, en torno a "no dejar a nadie atrás", la flexibilización del proceso educativo, la evaluación como mejora, la potenciación del uso didáctico de las tecnologías o el apoyo y refuerzo (Díez, et. al., 2020). Por lo anterior, se puede establecer que esta pandemia ha ocasionado que tanto docentes como estudiantes, hagan uso de las plataformas, aunado que se ha creado una sinergia entre ambas partes y una empatía, con la finalidad que ante tanto estrés y ansiedad que se ha generado, se pueda continuar trabajando en beneficio del alumnado.

En la investigación presentada por Arturo Barraza Macías en octubre de 2020, titulado "El estrés de pandemia (COVID 19) en población mexicana" se define al fenómeno estrés de pandemia como "Un estado psicológico producido por un proceso de adaptación" (p. 15). De acuerdo con la encuesta realizada se afirma que la población se encuentra en un nivel de estrés fuerte provocado por tres situaciones en particular: el riesgo de contagio, la desconfianza endémica en las instituciones nacionales y el distanciamiento social.

En el estudio realizado por Mancera, et. al., (2020) denominado “Pandemia: maestros, tecnología y desigualdad"se coincide sobre los límites que la pandemia ocasionó entre los niveles sociales de México, los cuales se pueden identificar fácilmente a través del censo del uso de tecnología e internet realizado por la ENDUTIH en 2019, donde muestra que el 56.4\% de los hogares mexicanos cuentan con acceso a internet.

Está información está ligada a la realidad del escenario educativo a distancia en donde los medios digitales son fundamentales para su aprovechamiento, quedando evidenciado que no todos los estudiantes tienen acceso. La desigualdad socioeconómica no debe pasar desapercibida, más bien, se debe tomar en cuenta al momento de crear métodos educativos de enseñanza o de utilizar nuevas plataformas en línea que tal vez no estén al alcance de todos.

En abril de 2020, el Instituto Internacional de la UNESCO para la Educación Superior en América Latina y el Caribe (UNESCO-IESALC) realizó un informe titulado "COVID-19 y educación superior: de los efectos inmediatos al día después" en el 
cual se examina cuáles están siendo los impactos inmediatos de la pandemia en el sector de la educación superior universitaria, tanto para los distintos actores como para las instituciones y el sistema en su conjunto, así como también se hace mención sobre la pérdida de contacto social y de las rutinas de socialización que forman parte de la experiencia cotidiana de un estudiante de educación superior. Afirman que el aislamiento asociado al confinamiento tendrá efectos en términos de equilibrio socioemocional que dejarán huella, en particular, en aquellos estudiantes con problemáticas preexistentes en este dominio y a los estudiantes más vulnerables que participan en programas de nivelación y apoyo, el aislamiento les golpeará aún más fuerte (UNESCO-IESALC, 2020).

La encuesta realizada en mayo de 2020, por la Asociación Internacional de Universidades (IAU Global Survey Report) titulada "El impacto del COVID-19 en educación superior alrededor del mundo" fue una técnica realizada por un periodo establecido del 25 de marzo al 17 de abril, en el que diversas personas (estudiantes, maestros o directores) de alguna institución de educación superior podían responder. Entre los resultados obtenidos, el 59\% de las instituciones fueron afectadas por la pandemia, apareciendo como una de las razones el cierre temporal de las escuelas que perjudicó al $79 \%$ de la población estudiantil. (Marinoni, G., et al., 2020). Gracias a este cuestionario internacional, se evidencia el impacto que la pandemia ocasionó en diferentes instituciones, ya sea de educación superior o cualquier nivel educativo.

Por último, se presenta a la Asociación Iberoamericana de Docencia Universitaria en México (AIDU México) la cual realizó esfuerzos importantes para coordinar entre agosto y septiembre del 2020 un foro virtual en el que actores educativos de Iberoamérica y Estados Unidos, compartieron su experiencia en el tema, al respecto expresa que:

Sin embargo, ahora, más que nunca, es necesario hacer conciencia de que en esta crisis sanitaria y económica mucha gente está sufriendo y muriendo. Apelemos a la solidaridad, la empatía y el afecto por encima de la indiferencia, construyamos una sociedad más justa e igualitaria, una sociedad que se caracterice por luchar contra la desigualdad, la exclusión y la discriminación (Rivera et.al. 2021, p. 9). 
Este llamado que realiza la AIDU es digno de recordar, indudablemente los estragos derivados de la pandemia de COVID 19 deben incidir en la sensibilización de la humanidad. Es difícil prever cuáles serán los escenarios, las subjetividades o las condiciones de la supervivencia que se narran en el mundo post- pandemia; sin embargo, celebramos que esta situación difícil nos ha permitido aportar, quizá con especulaciones, una narrativa potencial que nos convoca a aprender a ver, a disfrutar el mundo como es, y en fin, una narrativa que nos enseña que todo depende de nuestra capacidad colectiva de concebir, construir y reescribir el futuro de la educación: una tarea de todas y todos (Rivera, 2021).

\section{El Flipped Classroom en la educación superior}

Los retos que depara este vertiginoso y cambiante siglo XXI para estudiantes y docentes, obligan a estos actores a convertirse y formar profesionales que sean capaces de trabajar en los diferentes equipos multidisciplinarios sin importar el área laboral. Para lograr lo anterior, "será imprescindible fomentar el desarrollo de competencias fundamentales como la creatividad y el pensamiento crítico" (Carrió, et al., 2019, p.1).

En este sentido, Sola, et al. (2019) refiere que el aprendizaje activo y la inserción del estudiante como eje de su propio aprendizaje ha ocupado un lugar estelar en el aprendizaje. Es aquí en donde el método de aula invertida o Flipped Classroom, juega un papel fundamental permitiendo integrar el aprendizaje activo y las Tecnologías de la Información y Comunicación (TIC), lo que potencia el desarrollo de habilidades técnicas.

Plešec, R. (2017) menciona que el concepto de flipped classroom ha tenido diferentes etapas de evolución desde su aparición en 2006 con los pioneros del tema: Jonathan Bergmann y Aaron Sams. En un primer momento, se consideró que el maestro era el centro del aprendizaje y en él se encontraba la clave para la formación de los alumnos, mientras que los videos eran usados únicamente como apoyo en la proyección de contenido; sin embargo, en el último y tercer momento de este concepto, se posiciona al alumno como centro de su aprendizaje donde se buscan ahora estrategias educativas con el objetivo de que el conocimiento no solo se profundice sino también sea duradero.

Por otro lado, Abad-Segura et al. (2019) aseguran que el flipped classroom otorga una responsabilidad al alumno, implicando que reflexione sobre su 
aprendizaje y lo asocie con situaciones que optimizan su experiencia educativa, apoyándose en motivaciones e intereses personales y también en problemáticas o cuestiones que requieren de solución. Resulta relevante mencionar algunas de las características de este método: incremento del número de horas dedicadas al aprendizaje, uso de la tecnología e inversión de roles en el aula.

Miragrall y García Soriano (2016) refieren que la importancia del método de aula invertida, requiere compromiso por parte del docente y del discente. Es pertinente puntualizar que ambos, profesor y estudiante, deben contar con conocimientos avanzados en el manejo de las TIC, así como de los entornos virtuales.

El uso de metodologías activas como el método flipped classroom tiene como objetivo que el estudiante logre las competencias y desarrolle un aprendizaje autónomo, mediante el uso también de las TIC que a su vez le permitirán al docente ser el guía, el facilitador de los recursos y herramientas y por parte del estudiante lograr aprendizajes, estilos, ritmos y toma de conciencia en su educación (Ventosilla, et al., 2021).

Por tal motivo, es importante que los docentes puedan despertar el interés del alumno, mediante una perspectiva innovadora, creando nuevos escenarios de enseñanza-aprendizaje, fomentando un ambiente de armonía, mediante el empleo de diversas plataformas que se pueden descargar de manera gratuita, para no perjudicar la economía tanto del docente como la del discente.

Al amparo de las TIC, en los últimos años, han aparecido herramientas que sirven tanto para la enseñanza como para la presentación de contenidos. Estrategias educativas como las encuestas en línea (Google Forms ${ }^{\oplus}$ ) y los juegos de preguntas y respuestas (Kahoot ${ }^{\circledR}$ ) entre otras, están coligados en el proceso de enseñanza-aprendizaje (Chaiyo y Nokham, 2017 citado por Oliveira, et al., 2020). El aprendizaje de conceptos en un entorno incentivador, donde el alumnado se puede relacionar con su entorno permitiéndole conocer el mundo y desarrollar su creatividad, es una consecuencia de la incorporación de elementos lúdicos como parte del aprendizaje (Guardia, 2019 citado por Oliveira, et al., 2020).

Si bien es cierto, que para favorecer el aprendizaje de los estudiantes, se requiere entornos modernos y la implementación de herramientas didácticas innovadoras. Cabe mencionar que para que lo anterior cumpla su objetivo, Bretón de la Loza et al. (2019) menciona, que es de vital importancia partir de un diagnóstico situacional en el manejo de las tecnologías, conocimientos teóricos del área 
técnica del grupo de estudiantes en los que se pretende implementar el método de aula invertida. Partiendo de la premisa anterior, este autor sugiere aplicar un instrumento con dimensiones específicas que permita evaluar el nivel de conocimientos de los estudiantes $y$, así poder medir el avance en la obtención de conocimientos y de esta manera obtener los máximos beneficios del método de Flipped Classroom o aula invertida.

\section{METODOLOGÍA}

Esta investigación tiene un enfoque cualitativo, de tipo transversal descriptivo, con diseño no experimental. Sirvent (2007) desde su óptica establece, que el enfoque cualitativo es el que acerca al investigador a enfatizar la inducción analítica y con ello buscar la generación de una teoría que oriente a comprender e identificar verdades hipotéticas. En otras palabras, se trata de trabajar con pocos casos pero que al mismo tiempo permitan profundizar en el problema que afecta a una población. Por su parte Hernández, R. et al. (2014), mencionan que el enfoque cualitativo se elige cuando se pretende indagar cómo un grupo de individuos perciben y experimentan los fenómenos que los rodean. El mismo Hernández (2014) señala que el término diseño se refiere al "plan o estrategia concebida para obtener la información deseada con el fin de responder el planteamiento del problema" (Hernández, R. et al., 2014, p.128).

Se desarrolló en la Ciudad de San Francisco de Campeche, Campeche, México con la selección de cuatro instituciones de educación superior: tres de ellas ofertan estudios de licenciatura (dos públicas y una privada) y la cuarta institución ofrece posgrado (pública). El tipo y tamaño de muestra es por conveniencia. Para las instituciones públicas se seleccionaron todos los estudiantes matriculados en el primer semestre del ciclo escolar 2020-2021 Fase I (A) y para la escuela privada se eligieron los grupos del primer cuatrimestre y corresponde al ciclo escolar 20-22; alcanzando un total de 134 participantes (Tabla 1) de los cuales $108(80.59 \%)$ corresponden al nivel de licenciatura y 26 (19.4\%) a posgrado.

Para el proceso de recopilación de información, se aplicaron dos instrumentos: el primero una ficha de identificación de creación propia, integrada por catorce ítems: 1. Género, 2. Edad, 3. Trabaja además de estudiar, 4. Estado civil, 5. ¿Tiene hijos?, 6. ¿Con quién vive?, 7. Promedio del bachillerato, 8. Promedio obtenido 
en el primer semestre de licenciatura, 9. ¿Considera que ha sido fácil la adaptación a la nueva normalidad de trabajo en línea y a distancia?, 10. ¿Cuál ha sido la mayor complicación en las clases en línea?, 11. ¿Cómo ha influido la modalidad virtual en su desempeño académico?, 12. ¿Cómo considera que ha sido el aprendizaje obtenido en este ciclo? 13. ¿Durante este escenario de clases virtuales, considera usted que ha presentado alguna alteración de ansiedad, pérdida o aumento de apetito? y 14 . Describa las afectaciones socioemocionales que ha presentado durante este ciclo escolar que ha concluido. Este instrumento se utilizó para la recopilación de datos de identificación de los sujetos de estudio; para tener el dato preciso por institución educativa fue necesario crear cuatro copias, generar un link, el cual se compartió a cada responsable de escuela y a su vez lo hizo llegar a sus estudiantes, dando el respectivo seguimiento a las respuestas. El segundo instrumento se titula:Test de estrés laboral, adaptado del cuestionario de problemas psicosomáticos (o CPP) realizado en colaboración con el Gobierno de México y el IMSS, el cual mide los síntomas asociados al estrés con la siguiente valoración: Sin estrés (12-24), Estrés leve (36), Estrés medio (48), Estrés alto (60) y Estrés grave (72). Este instrumento sólo se aplicó a quienes expresaron en el cuestionario uno, que trabajaban; los dos instrumentos fueron construidos en formulario de la aplicación de la Suite de Google, el link fue compartido directamente al correo del estudiante, solicitándoles a los integrantes del equipo de investigación, dieran seguimiento a las respuestas de los estudiantes.

Todos los formularios fueron realizados, concentrados y analizados por el equipo técnico responsable de esta actividad.

\section{Recolección de datos}

Como parte del compromiso de ética en la investigación, previo al inicio de las actividades derivadas el estudio, se presentó a los grupos de participantes el protocolo, integrando los aspectos específicos a realizar, para su conocimiento y consideraciones, especificando que los resultados serían utilizados para fines estadísticos, también se les explicó que tenían la libertad de retirar su participación, sin ningún tipo de represalia o sanción; otro aspecto ético fue la confidencialidad debido a que en los formularios aplicados, en ningún momento se solicitó información personal, que se considere sensible o confidencial. 


\section{Análisis de los datos}

Para el análisis de los datos se utilizó una hoja electrónica de Excel, la cual está integrada en la herramienta Google Forms. Una vez respondidos y enviados ambos instrumentos, la herramienta genera en forma automática una hoja de cálculo electrónico en donde indica cuantos participantes respondieron la pregunta indicando el porcentaje de participación.

Las cuatro instituciones de educación superior incluidas en este estudio han enfrentado de forma muy particular los efectos de la pandemia ocasionada por el virus SARS-CoV-2, a continuación, se describen los hechos ocurridos en cada caso. La Universidad Autónoma de Campeche (UACam), institución con más de 55 años en el ámbito de la educación superior y media superior en el estado de Campeche, México, en marzo de 2020 en respuesta a la declaración de contingencia sanitaria por COVID 19, instruye cerrar las instalaciones y migrar las clases

\section{Tabla 1. Características de la población}

\begin{tabular}{|c|c|c|c|}
\hline INSTITUCIÓN & TIPO & OFRECE & $\begin{array}{l}\text { PARTICIPANTES } \\
\text { ESTUDIANTES }\end{array}$ \\
\hline $\begin{array}{l}\text { 1. Universidad Autónoma } \\
\text { de Campeche (UACam) }\end{array}$ & Público & $\begin{array}{c}\text { Licenciatura } \\
\text { en Gerontología }\end{array}$ & $39(29.10 \%)$ \\
\hline $\begin{array}{l}\text { 2. Benemérito Instituto } \\
\text { Campechano (BIC) }\end{array}$ & Público & $\begin{array}{c}\text { Licenciatura } \\
\text { en Gastronomía }\end{array}$ & $46(34.32 \%)$ \\
\hline $\begin{array}{l}\text { 3. Universidad Vizcaya } \\
\text { de las Américas (UVA) }\end{array}$ & Privado & $\begin{array}{l}\text { Licenciatura } \\
\text { en Nutrición }\end{array}$ & $23(17.16 \%)$ \\
\hline $\begin{array}{l}\text { 4. Universidad Pedagógica } \\
\text { Nacional, Unidad } 041 \text { (UPN) }\end{array}$ & Público & $\begin{array}{c}\text { Maestría en } \\
\text { Gestión Educativa }\end{array}$ & $26(19.4 \%)$ \\
\hline
\end{tabular}

Fuente: Elaboración propia, 2021 
a la modalidad virtual, solicitando a los docentes flexibilidad hacia los estudiantes. En este sentido, la asistencia ya no fue un requisito para acreditar la unidad de aprendizaje. Por lo que los estudiantes podían o no asistir a las sesiones académicas virtuales programadas.

En cuanto a la forma de evaluación, la aplicación del examen departamental estandarizado (EXADES) que por tradición se administraba en todos los programas educativos y tenía asignado el $40 \%$ del valor total de la calificación de cada examen parcial, fue suspendido, siendo sustituido por el uso de un formulario de Google Suite, ahora con un valor de $10 \%$ de la calificación. Bajo estas condiciones, concluye la Fase 2 del ciclo escolar 2019-2020. El siguiente semestre, Fase 1 del ciclo escolar 2020-2021 fue diferente, las asistencias ya son consideradas para efectos de acreditación de las asignaturas, ahora se pasa lista de asistencia en cada sesión, lo que obliga a los estudiantes a asistir a las sesiones académicas virtuales y, el docente, adquiere elementos de ponderación para otorgar o no una nota promocional.

Para enfrentar este reto, las autoridades de la UACam contrataron a una empresa de consultoría para que diseñara e implementara una estrategia que permitiera dotar a los docentes de información actualizada y útil para enfrentar este reto. De esta manera, surge el Modelo de Acompañamiento, el cual consistió en aplicar un programa de capacitación integrado por tres talleres: Uso de herramientas para el modelo de acompañamiento, Diseño de actividades con recursos en línea, y Diseño estandarizado de espacios de aprendizaje, todos en línea con actividades autoadministrables y dos sesiones síncronas para resolver dudas.

Como parte de la estrategia, se seleccionó a los docentes que serían capacitados como facilitadores, este grupo de profesores fueron los primeros en conocer la estructura, materiales y trabajaron en su integración, posteriormente se les asignó el grupo de compañeros docentes con quienes colaborarían. Cada uno de los tres cursos, tuvo una duración de cinco días hábiles, los tiempos estuvieron muy ajustados dado que el semestre estaba por iniciar, se encontró resistencia a la capacitación, los docentes que no acreditaron, fueron comisionados nuevamente en la Fase 2, ciclo escolar 2020-2021, siendo evidente el compromiso institucional hacia los docentes. Resulta importante mencionar que para algunos docentes la capacitación no fue de su interés y no participaron.

Después de la fase de capacitación, se diseñaron las políticas de operación del servicio académico, exigiéndose que las horas síncronas fueran mínimo el 50\% 
de lo asignado, es decir, si el docente tiene cuatro horas/semana/mes (HSM) solo debe programar dos horas a la semana para las sesiones académicas en línea y dos horas a la semana en las cuales otorgará asesoría y revisión de evidencias, dado que el docente ya tiene grabado en video el tema acorde al contenido temático del programa de la unidad de aprendizaje que imparte, además, ahora el profesor deberá llenar un nuevo formato denominado tabla de actividades por cada subcompetencia, y debe crear una sala para su unidad de aprendizaje (materia) en Google Chat e invitar a sus estudiantes. Es importante mencionar, que la UACam desde años anteriores ya había implementado la plataforma educativa Classroom, y trabajó en la certificación de sus docentes, toda esta labor le valió para que, en septiembre de 2019 , se le otorgara la certificación Google Reference University, convirtiéndose así en un referente nacional.

Los estudiantes han expresado su deseo de volver a las aulas de forma física, sobre todo en el área de la salud, al ser importante la práctica clínica, además de las limitaciones y complicaciones que han tenido en sus casas al carecer de computadoras, una conexión eficiente de internet y un área de estudio que proporcione privacidad que permita concentrarse para poder estudiar. Es de vital importancia puntualizar que la carencia de espacios físicos en los hogares compromete la interacción y relación estudiante-docente lo que afecta la recepción y comprensión por parte del alumno y la transmisión de conocimiento por parte del docente (procesos de enseñanza - aprendizaje).

Por otro lado, debido a la falta de empleos de los padres de familia, muchos de los estudiantes se han visto en la necesidad de trabajar en sus ratos libres para contribuir a la economía familiar. También los docentes han debido adaptarse a esta nueva normalidad, teniendo que adquirir una computadora con cámara, un buen micrófono, audífonos, mejorar su señal de internet, adaptar un espacio en su casa, cuidar los ruidos externos mientras están en sesión. Un aspecto importante, es que los docentes han ido perdiendo su privacidad pues ahora el espacio de su casa es público, todos sus estudiantes lo conocen, además deben estar pendientes de atender las dudas que los estudiantes escriban en el chat de su sala virtual de aprendizaje. Se reconoce que el pago de los docentes se mantuvo, con todas las prestaciones de ley y todos los beneficios signados en el contrato colectivo de trabajo, incluyendo el otorgamiento de los procesos de promoción y entrega de definitividad, así como el programa de estímulo al desempeño del personal docente. 
El Benemérito Instituto Campechano (BIC), con 161 años como referencia educativa y cultural, durante la pandemia sufrió cambios muy drásticos, tanto para directivos, docentes, administrativos y alumnos, debido a que se continuó con el trabajo con los estudiantes. Esto implicó un fuerte impacto para los maestros, ya que se tuvo que implementar estrategias para dar las sesiones de clases a través del uso de plataformas como Moodle, para poder impartir las clases virtuales, que ya se había implementado pero que no era de carácter obligatorio su uso, aunado al empleo del WhatsApp y el correo electrónico.

Sin embargo, al concluir el semestre febrero - julio de 2020, el uso de las plataformas ya no era una opción, sino que era una de las herramientas indispensables para la labor diaria. Lo anterior significó una gran presión pues en menos de tres meses el $90 \%$ de los directores y docentes del BIC, se tuvieron que certificar en Google por Suite, Nivel 1, provocando estrés y ansiedad según los comentarios entre los mismos maestros.

Para los alumnos, también ocasionó altos niveles de estrés, al no contar en su mayoría con el equipo (computadora), ni con la conectividad (internet) para sus clases virtuales, tomando en consideración que se tienen estudiantes de comunidades rurales, con el acceso a internet complicado o nulo.

La situación descrita causó mucho impacto, a pesar que se tiene la flexibilidad para apoyar a los alumnos en la entrega de tareas, en las asesorías externas para acompañarlos en sus procesos de aprendizaje, no deja de causar conflicto en el estado de ánimo de muchos de ellos. Cabe mencionar que, aunado a lo anterior, algunos estudiantes también trabajan para poder pagar sus estudios y con esta pandemia la mayoría perdió su empleo, incluyendo el de sus padres, estas son algunas de las razones por las que al menos el 5\% decidió dejar de estudiar. De igual forma, tanto docentes como discentes, aprendieron a trabajar en el uso de las plataformas durante el semestre septiembre 2020 a enero 2021, las más utilizadas fueron Google Classroom, Richmond y Duolingo, estos dos últimos implementadas para el área de idiomas, para facilitar sus sesiones virtuales. En momentos de la globalización, la tecnología ha impuesto nuevas dinámicas en todos los campos, que han permeado ampliamente en el área de la educación, brindando diversos soportes fortalecedores del quehacer pedagógico del docente, ofreciendo un ramillete de posibilidades educativas con las cuales se puede contar en el ciberespacio (Bernal, S. y Ramírez, A., 2020). 
Si bien es cierto que la labor docente ha incrementado, dado que el BIC, cuenta con una certificación en la ISO 21001:2018, lo que ocasiona que actualmente se tiene que evidenciar todo el trabajo realizado en las aulas virtuales (entrega de los programas de la unidad de aprendizaje, los criterios de evaluación, las tareas, asesorías, las sesiones de clases se graban, por mencionar algunas), para garantizar la calidad educativa, también se reconoce que la percepción de los salarios ha continuado puntualmente con todas las prestaciones de ley. La Universidad Vizcaya de las Américas (UVA) Campus Campeche, desde su apertura en el mes de septiembre del 2018, los docentes y alumnos fueron capacitados en el uso de dos plataformas: Sesweb y Classroom, pero solo eran utilizadas para subir calificaciones, dejar indicaciones y entregar tareas. Los alumnos fueron evaluados con carpeta de evidencia, examen y participación; desde que inició la suspensión de labores escolares el 20 de marzo para escolarizado y 21 de marzo para sabatino. Los docentes afrontan el primer reto: el económico, al estar en un sistema privado, se paga de acuerdo a las horas frente al grupo, es decir que se trabajó hasta el 8 de mayo, todo un mes sin pago; el afrontamiento de la última quincena de marzo fue complicada al pagarse solo cuatro días (17, 18,19 y 20), significando que la primera quincena que se cobró fue a finales del mes de mayo (es decir la segunda quincena de mayo del año 2020); siendo una situación difícil y de gran afectación económica para aquellos docentes para quienes esta es su única fuente de empleo.

El 14 de mayo del 2020, la autoridad académica convocó a una reunión vía Meet (aplicación de videollamada generada por Google), en la cual se explica a los docentes sobre el retorno a las clases de manera virtual, se expuso de manera extensa sobre el dominio de la plataforma Meet. Donde los docentes cumplían su máximo nivel de horas estipulada por Rectoría, 17 horas frente al grupo, en cuatrimestre que corresponde mayo-agosto se reportó sin novedad, porque se cumplió con los programas; sin embargo para el mes de agosto la universidad evaluó los servicios docentes con los estudiantes, donde se manifestó demasiado tiempo de clases, el pago de las colegiaturas mantenían el mismo costo en mensualidad e inscripción, docentes rígidos y exigentes, falta de empatía, nulo uso de las TIC y calificaciones deficientes o bajas.

A partir del regreso a clases el docente tiene que cumplir con requisitos semanales, subir evidencias en el portal Sesweb del inicio del cuatrimestre cargar planeaciones, evidencias fotográficas (ya que las pruebas funcionan como checar 
asistencia), asistencias del alumno (semanalmente), vía Drive (semanal), cargar los temas semanales, generando mucha ansiedad, debido a que, si hay olvido, no se genera el pago adecuadamente.

En el mismo mes de agosto 2020, a los docentes se les indica que tienen que emplear las herramientas digitales, mediante una serie de diapositivas, pero, no se les facilitó explicación sobre el uso de las mismas, significando un reto adicional para los docentes y alumnado; de las cuales se agruparon en páginas y aplicaciones generadoras de audiovisuales, didácticas (sopas de letras, crucigramas, vinculación, entre otros), blogs académicos, wikis (glosarios), creador de mapas mentales y conceptuales animados. La mayoría de las aplicaciones se encuentran en idioma inglés; pero con traductor en las pestañas se podía resolver. En el mismo mes se les notifica que las horas en clases disminuirían una hora en cada asignatura, los docentes disminuyen la rigidez y se tuvo que ser más empático por cuestiones diversas, tal como, despido de los padres y la transición de estudiante-empleado para costear sus colegiaturas. La UVA campus Campeche sigue implementando becas internas, así como las ofrecidas por H. Ayuntamiento, y el Estado a través de la Fundación Pablo García, adicional a la promoción por pagos anticipados, todo ello con la finalidad de mantener la matrícula.

La Universidad Pedagógica Nacional (UPN), Unidad 041 Campeche, es una institución de amplia trayectoria, de reconocimiento nacional y muy tradicional, en donde se privilegia el trato cercano en la modalidad presencial, se carecía de la obligatoriedad del uso de plataformas virtuales, se tuvo Moodle en algún momento, cuando el presupuesto lo permitió; con el escenario de la pandemia, surge la necesidad de la transición a la virtualidad, cada asesor pedagógico encontró las herramientas para estar en comunicación con sus estudiantes, desde WhatsApp, correo electrónico, así se terminó el semestre de marzo a agosto de 2020, al continuar con la misma situación de distanciamiento social, ya en el semestre septiembre 2020 a febrero 2021, se solicitó el uso de la plataforma Classroom, sin contar con la implementación de una estrategia de capacitación dirigida a los asesores pedagógicos. El personal docente continuó con la percepción en relación con su ingreso económico y permaneció trabajando desde su casa, sólo el personal administrativo estaba obligado a la asistencia presencial, aplicando las medidas sanitarias indicadas. 


\section{RESULTADOS}

De las cuatro instituciones estudiadas se observa que en todas existe evidencia de una estrategia implementada por parte de la autoridad, sin embargo, la Universidad Autónoma de Campeche es la que presenta un modelo integral pensando en los docentes y en los estudiantes.

De los resultados del formulario de identificación se presentan los resultados siguientes:

En la figura 1 se realiza la comparación de calificaciones solo de las tres instituciones que ofrecen educación superior, contrastando el promedio obtenido en el bachillerato y lo obtenido en el primer semestre.

Es evidente el incremento del promedio en el primer semestre de licenciatura, sobre todo en el rango de nueve a diez, interpretándose que las clases en línea han sido de beneficio para su aprovechamiento escolar. Aquí es importante

\section{Figura 1. Comparativo de calificaciones}

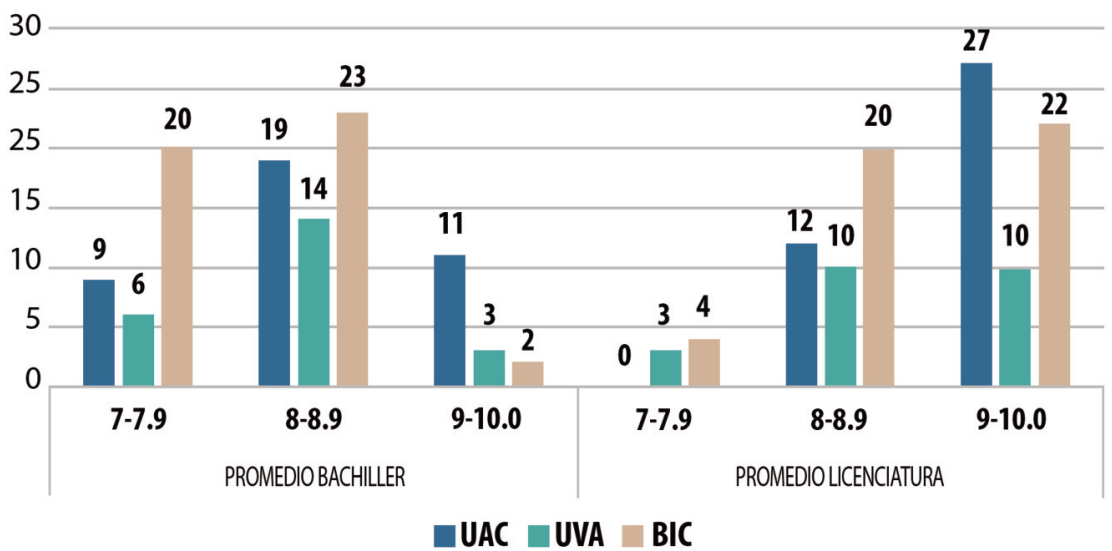

Fuente: Elaboración propia, 2021 
aclarar la razón por la cual se ha omitido el dato de la UPN, esto es debido a que el semestre concluye apenas en febrero de 2021 y no fue posible tener esta información durante la aplicación del instrumento.

En la figura 2, se responde el ítem siguiente: ¿Considera que ha sido fácil la adaptación a la nueva normalidad de trabajo en línea y a distancia? se presenta que 90 estudiantes (67\%) no se han adaptado a la nueva normalidad y 44 (33\%) expresaron que sí se han adaptado.

Es interesante observar que a pesar de que la diferencia no es significativa en cuanto a la adaptación, la nueva normalidad sí les ha servido para incrementar su promedio.

En la figura 3, responde al ítem: ¿Durante este escenario de clases virtuales, considera usted que ha presentado alguna alteración de ansiedad, pérdida o aumento de apetito? 42 estudiantes (31\%) señalaron no percibir ninguna alteración y 92 (69\%) expresaron haber percibido alteraciones.

\section{Figura 2. Adaptación a la nueva normalidad}

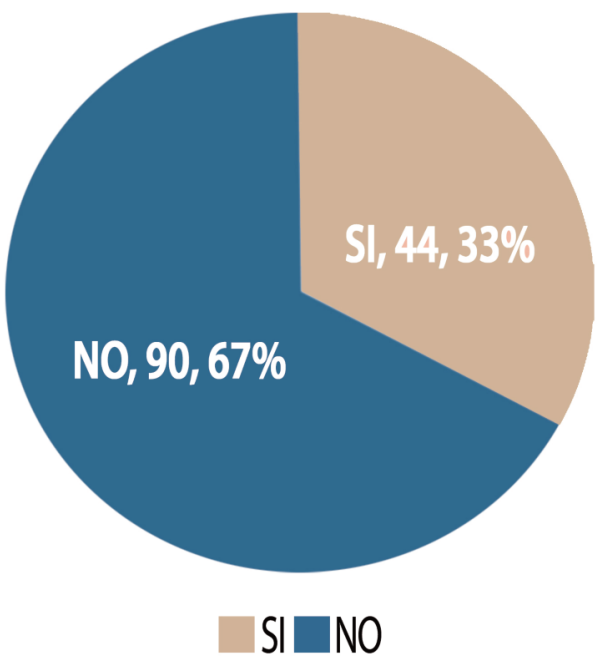

Fuente: Elaboración propia, 2021 


\section{Figura 3. Autopercepción de ansiedad}

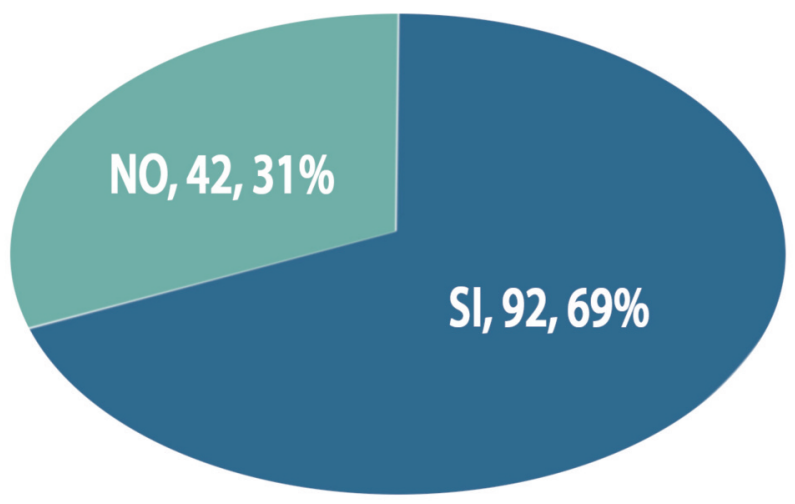

$\square \mathrm{SI}$ NO

Fuente: Elaboración propia, 2021

Cerca del $70 \%$ ha sentido ansiedad por esta modalidad en línea, este resultado es significativo, evidencia el esfuerzo que están realizando por mantener y mejorar su promedio.

En la figura 4, responde al siguiente ítem ¿Cómo ha influido la modalidad virtual en su desempeño académico?, con las opciones de: se ha mantenido, disminuido o incrementado, solamente 10 (7\%) expresaron que ha disminuido. El 52\% señaló que ha mantenido su desempeño a pesar de la modalidad a distancia.

En la figura 5, se responde al ítem ¿Cómo considera que ha sido el aprendizaje obtenido en este ciclo?, el 50.36\% (69) señaló que bueno y el 7.29\% (10) expresaron que ha sido excelente.

Es evidente el esfuerzo realizado por docentes y estudiantes, en este escenario de nueva normalidad, ha valido la pena la suma de talentos.

En los datos obtenidos en el formulario del Test de estrés laboral, se encontró que ninguno se ubica en el nivel grave, en el alto solo dos estudiantes. En la 


\section{Figura 4. Desempeño académico}

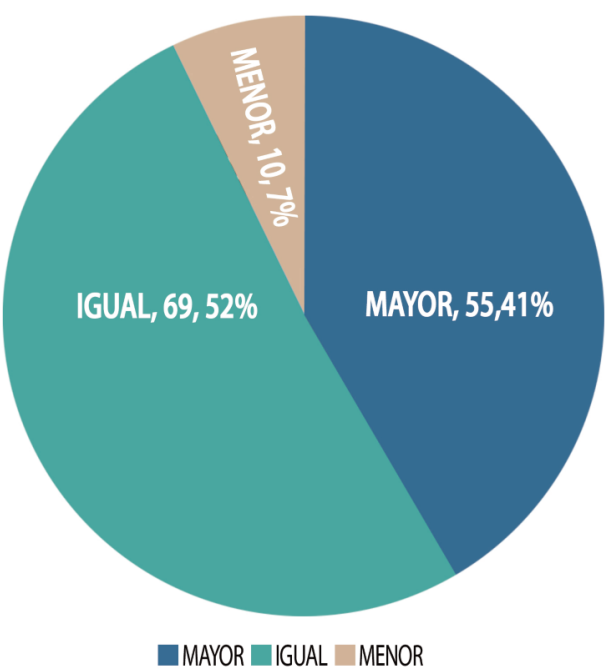

Fuente: Elaboración propia, 2021

\section{Figura 5. Consolidación del aprendizaje}

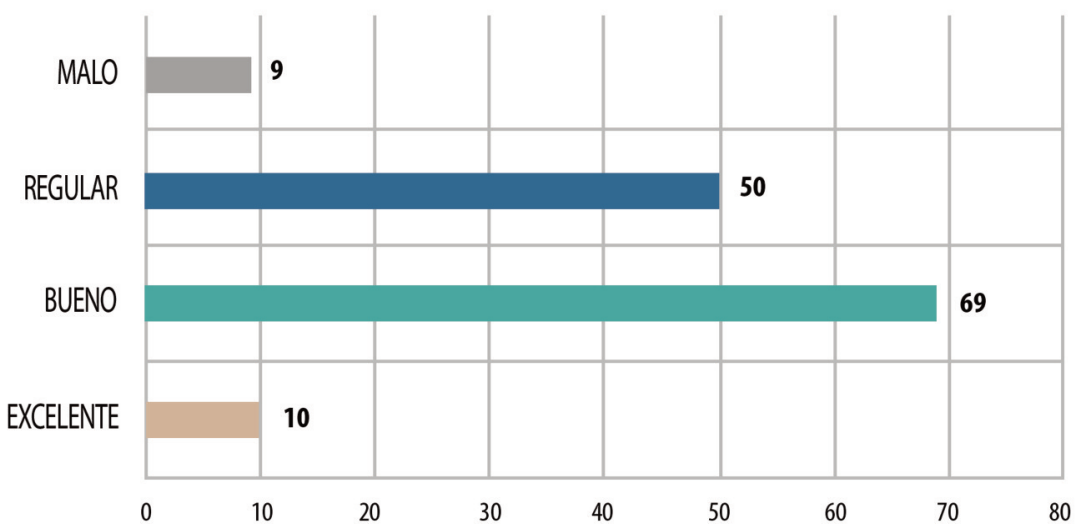

Fuente: Elaboración propia, 2021 
tabla 2, se encuentra especificado la cantidad de estudiantes que manifestaron que trabajan además de estudiar, así como el resultado del instrumento que mide el estrés laboral.

Sin estrés (12-24), Estrés leve (36), Estrés medio (48), Estrés alto (60) y Estrés grave (72), Se observa que ninguna de las cuatro instituciones logró alcanzar el $80 \%$ en la resolución del formulario, solo UACam estuvo cerca de lograrlo. De los 61 participantes que expresaron estudiar y trabajar, solo respondieron 37 (60.65\%).

El instrumento de estrés laboral es presentado en la figura 6 , resultando que ninguno de los sujetos de estudio se encuentra en el nivel grave, el $5.40 \%$ se ubica en alto.

En la figura 6, se indican los niveles de estrés laboral expresado por los estudiantes, se identificó existencia de mayor incidencia en el nivel sin estrés.

\section{DISCUSIÓN Y CONCLUSION}

En este punto es necesario precisar que hoy día, el equilibrio del desarrollo socioemocional, es uno de los componentes estructurales que le dan sentido a la vida del ser humano. La influencia generada por factores medio ambientales, naturales y provocados, en este caso la pandemia, desencadenan comportamientos atípicos que se presentan como síntomas psicosomáticos; una de ellas es la ansiedad, hasta convertirse en patologías y/o enfermedades orgánicas.

Con base en los instrumentos aplicados en esta investigación se afirma que, el comportamiento humano, es un conjunto de complejidades en procesos de interacción social, lo cual genera a su vez diversidad de dificultades. De éstas, se desprenden las conductas que se han observado a partir del confinamiento derivado de la pandemia.

En cada institución participante en esta investigación, se aplicó el mismo número de instrumentos, obteniendo resultados diferenciados; las condiciones medio ambientales jugaron un rol fundamental. Si bien se puso atención a cuatro aspectos señalados como son: adaptación a la nueva normalidad, autopercepción de ansiedad, desempeño académico y consolidación del aprendizaje, los resultados, indican que aún existe resistencia a la nueva normalidad, aún compleja y difícil de discernir.

En tanto que, en la autopercepción de ansiedad, es preciso mencionar que con o sin pandemia, existe una marcada ansiedad en los estudiantes, cuando se so- 
Tabla 2. Concentrado de estudiantes que trabajan

\begin{tabular}{|c|c|c|c|c|}
\multicolumn{2}{|c|}{ IES } & TRABAJAN & \multicolumn{2}{c}{ RESOLVIERON } \\
\hline UACAM & 9 & $7(77.77 \%)$ & $\operatorname{Sin}(0)$, Leve (3), Medio (3), Alto (1) \\
\hline BIC & 17 & $9(69.23 \%)$ & Sin (7), Leve (1), Medio (1) \\
\hline UVA & 9 & $3(33.33 \%)$ & Sin (1), Leve (1), Medio (1) \\
\hline UPN & 26 & $18(52.94 \%)$ & Sin (11), Leve (2), Medio (2), Alto (1) \\
\hline
\end{tabular}

Fuente: Elaboración propia, 2021

Figura 6. Determinación de nivel de estrés laboral en estudiantes

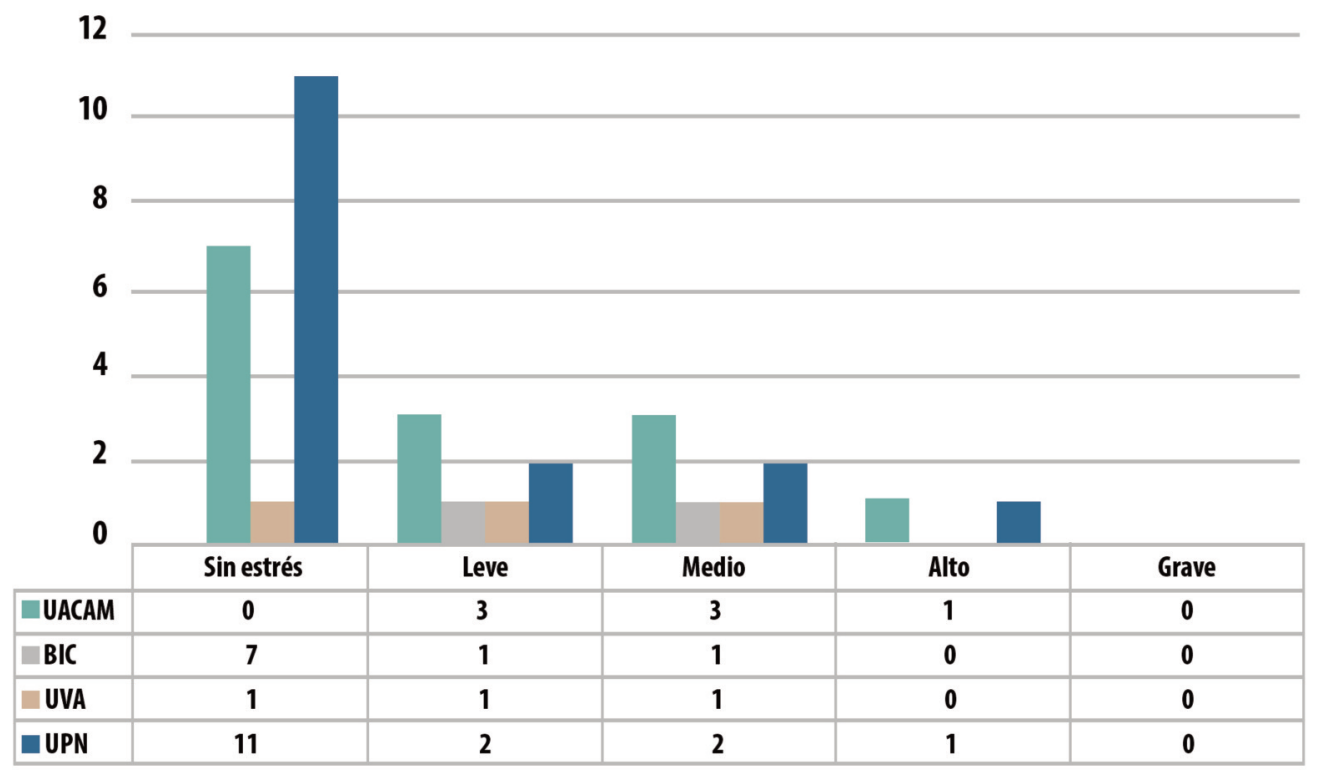

Fuente: Elaboración propia, 2021 
meten a las presiones por cumplir con los compromisos escolares. En este sentido, el rol de la escuela y su vinculación con los estudiantes, es un generador natural de ansiedad. En el desempeño académico, destacan los resultados que, no hay ninguna variación y que la pandemia, no marcó diferencia alguna. En tanto que son buenos, los resultados relacionados con el aprendizaje de los alumnos.

Es un hecho que el problema pandemia-contingencia-educación en línea, tomó por sorpresa a la sociedad, a la comunidad escolar y a los padres de familia, exigiendo afrontar situaciones inéditas y que han obligado a tomar decisiones alternas a la educación escolarizada.

Seguidamente se presenta la relación que tiene la pandemia con los diferentes aspectos analizados:

\section{Pandemia, Perspectivas}

También se pueden mencionar también, otros estudios como los que ha realizado la IESALC-UNESCO acerca de la pandemia, destaca: COVID-19y educación superior: De los efectos inmediatos al día después (2020), en el que se señalan las perspectivas educativas que se deberían considerar ante la presencia del COVID y después de dicho fenómeno. Sin embargo, en este escrito se consideró, que la pandemia estaría en la sociedad poco tiempo; tiene casi ya 18 meses y, no desaparece, por ello estamos construyendo propuestas alternas.

\section{Pandemia y estrés}

Otro estudio vinculado a la cuestión socioemocional, específicamente al estrés, es el que menciona Barraza, (2020), en: El estrés de pandemia (COVID 19) en población mexicana, en el que enfatiza, cómo lo incierto y el temor, se han apoderado de la existencia de personas y grupos; lo cual ha generado comportamientos somáticos, patológicos y comportamentales, teniendo como consecuencias desenlaces violentos $\mathrm{y} / \mathrm{o}$ fatales.

\section{Pandemia: Ética y política}

Las cuestiones éticas y políticas que la pandemia ha generado no sólo están presentes en los temas y programas de la salud, sino en la educación. Donde el sujeto social en la educación, tiene un rol relevante. Esto lo plantea atinadamente Enrique Dussel, (2020), en: La pandemia con Enrique 
Dussel. Ética y política, para atender de manera pertinente las necesidades no solo de uso de las TIC, sino de la apropiación, desde casa, de contenidos que antes de la pandemia se revisaban en el aula.

En síntesis, la contingencia sanitaria ha generado éstas y otras reflexiones, a la par de distintas estrategias; no solo de políticas públicas, sino de planeación, hasta llegar a la aplicación didáctica tanto en la modalidad escolarizada como virtual, de lo que hoy día debemos entender, lo que significa vivir en, con la pandemia y desde casa ante procesos virtuales de enseñanza, aprendizaje y evaluación. La pandemia, provocó una transformación radical de la cotidianidad mundial y de los sistemas educativos en particular. En este sentido, existe la necesidad de continuar adaptando lineamientos y contenidos de planes y programas a contextos virtuales y /o digitales, siendo este un desafío para los agentes escolares (alumnos, docentes, administrativos, padres de familia, entre otros). Las TIC y sus diversas dimensiones siguen siendo un enorme desafío, para contextos de desarrollo medio, como el caso de México.

Finalmente, en materia de salud mental y sus derivaciones en los alumnos y preparación docente en torno a la enseñanza en línea, el Estado mexicano tiene que implementar estrategias y políticas públicas educativas que impulsen otras opciones no escolarizadas de la educación virtual para afrontar tiempos de crisis.

\section{REFERENCIAS}

Abad-Segura, E. y González-Samar, M.D. (2019). Análisis de las competencias en la educación superior a través de flipped classroom. Revista Iberoamericana de Educación 80(2), pp. 29-45. https://doi.org/10.35362/rie8023407

Barraza Macías, A. (2020). El estrés de pandemia (COVID 19) en población mexicana. Universidad Pedagógica de Durango. México. http://www.upd.edu.mx/PDF/Libros/Coronavirus.pdf

Borgobello A., Madolesi M., Espinosa A., Sartori M. (2019). Uso de TIC en prácticas pedagógicas de docentes de la Facultad. Revista de Psicología, 37 (1), pp. 279-317. https://doi.org/10.18800/psico.201901.010 
Bretón de la Loza, M.M. Guerrero Ceh, J.G, Zúñiga Hernández, O. Y., Cervantes Ramírez, L.T., Garduño Díaz, S.D., Carranco Gómez, S. \& Terrazas Meraz, M.A. (2019). Validation of a questionnaire for the evaluation of clinical competencies in nutrition. International Journal of New Education. 4. pp.56-74. https://doi.org/10.24310/JNE2.2.2019.7451

Carrió, M., Moyano, E., Rodríguez, G., Sánchez, R. (2019). Flipped Classroom para desarrollar la creatividad en el área de medicina. Revista Observatorio de Innovación Educativa.

Díez Gutiérrez, E.J. y Gajardo Espinoza, K. (2020). Políticas educativas en tiempos de Coronavirus: la confrontación ideológica en España. Revista Internacional de Educación para la Justicia Social, Vol. 9 (3e), pp. 83-101. https://doi.org/10.15366/riejs2020.9.3.005

Dussel, E. (2020). La pandemia con Enrique Dussel. Ética y política | Video. https://aristeguinoticias.com/1604/mexico/2020-la-pandemia-con-enrique-dussel-etica-ypolitica-en-vivo-2/

Estrada-Araoz, E. G., Gallegos-Ramos, N. A., Mamani-Uchasara, H. J., y HuayparLoayza, K. H. (2020). Actitud de los estudiantes universitarios frente a la educación virtual en tiempos de la pandemia de COVID-19. Revista Brasileira de Educação do Campo, 5, e10237, p. 16. http://dx.doi.org/10.20873/uft.rbec.e10237

García Ruiz, J. y Kairuz Correa, J. (2020). COVID-19 Acciones globales frente al cierre de escuelas durante una pandemia. Universidad de Los Andes, Colombia.

https://uniandes.edu.co/sites/default/files/asset/document/covid-19acciones_globales-cierre-escuelas.pdf

Hernández Sampieri, R., Fernández Collado, C., Baptista Lucio, P. (2014). Metodología de la investigación. México, D.F. Sexta Edición, McGrawHill Education.

Instituto Internacional de la UNESCO para la Educación Superior en América Latina y el Caribe (UNESCO IESALC), (2020). COVID-19 y educación superior: de los efectos inmediatos al día después. Análisis de impactos, respuestas políticas y recomendaciones. https://www.iesalc.unesco.org/wpcontent/uploads/2020/05/COVID-19-ES-130520.pdf 
Instituto Internacional para la Educación superior en América Latina y el Caribe, (UNESCO IESALC), (2020). Encuesta global de la IAU sobre el impacto del COVID-19 en la educación superior alrededor del mundo. https://www.iesalc.unesco.org/2020/05/27/encuesta-global-de-la-iau-sobre-el-impacto-delcovid-19-en-la-educacion-superior-alrededor-del-mundo/

Instituto Mexicano del Seguro Social. (s. f.). Test estrés laboral. http://www.imss.gob.mx/sites/all/statics/salud/estreslaboral/Test-Estres-Laboral.pdf

Mancera Corcuera, C., Serna Hernández, L., y Barrios Belmonte, S. (2020). Pandemia: maestros, tecnología y desigualdad. NEXOS. https://educacion.nexos.com.mx/?p=2286

Miragall, M. y García-Soriano, G. (2016). Transformando una clase del grado en psicología en una flipped classroom. @TIC. Revista d'Innovació Educativa, 17, pp.21-29. https://doi.org/10.7203/attic.17.9097

Marinoni, G., van't Land, H. y Jensen, T. (2020). The impact of covid-19 on higher education around the world. International Association of Universities (IAU). Global Survey Report. https://www.iauaiu.net/IMG/pdf/iau_covid19 and_he_survey_report_final_may_2020.pdf

Oliveira Jardim, E., Lo-lacono-Ferreira, V. G., Arroyo López, M.R., Linares Pérez, N. (2020). Desarrollo y evaluación de habilidades y competencias en el ámbito de estudios relacionados con la sostenibilidad. El uso de las TICs como medio de instrucción. En: Roig-Vila, R. et al. Redes de investigación e innovación en docencia universitaria. Volumen 2020, pp. 849-859. Universidad de Alicante. ISBN 978-84-09-20703-9. http://hdl.handle.net/10045/109964

Plešec Gasparič, R., (2017). Jonathan Bergmann y Aaron Sams, flipped learning: gateway to student engagement, Sociedad Internacional para la Tecnología en la Educación: Eugene, Oregon y Washington, DC, 2014; ISBN 978-1-56484-344-9. Center for Educational Policy Studies Journal 7(3), pp. 173-176. https://www.cepsj.si/index.php/cepsj/issue/view/20/7-3

Rivera Morales, A. Heros Rondenil, M. D. L. y Hernández Mondragón, A. R. (2021). Vivir la docencia en tiempo de pandemia: experiencias en videocharlas de los actores de educación media superior y superior. Editorial Arquinza. https:/elibro.net/es/ereader/uacam/170245?page=9 
Sirvent, M.T., (2007). Investigación y estadística educacional I: el proceso de la investigación. Buenos Aires, Argentina. Tercera edición.

http://materiales.untrefvirtual.edu.ar/documentos extras/01305 princ de metod y sistem d e_la_pract i/Unidad1b/ElProcesodelnvestigacionParte1.pdf

Sola Martínez, T., Aznar Díaz, I., Romero Rodríguez, J.M., Rodríguez García, A.M. (2019). Eficacia del método Flipped Classroom en la universidad: metaanálisis de la producción científica de impacto. Revista Iberoamericana sobre Calidad, Eficacia y Cambio en Educación 17(1), pp. 25-38.

Tourón, J. y Santiago, R. (2015). El modelo flipped learning y el desarrollo del talento en la escuela. Revista de Educación, 368, pp.196-231. https://doi.org/10.4438/1988-592X-RE-2015-368-288

Ventosilla Sosa D. N., Santa María, H. R., Ostos De La Cruz, F. y Flores Tito, A. M. (2021). Aula invertida como herramienta para el logro de aprendizaje autónomo en estudiantes universitarios. Propósitos y Representaciones 9(1), e1043. DOI: http://dx.doi.org/10.20511/pyr2021.v9n1

\section{BIONOTAS}

Jaqueline Guadalupe Guerrero. Profesora investigador de tiempo completo en la Universidad Autónoma de Campeche. Doctorado en Ciencias de la Educación, Maestría en Gerontología, Maestría en Educación Superior, Licenciatura en Literatura. Publicación de artículos y capítulos de libro, Dirección de Tesis de doctorado y licenciatura.

Correo electrónico: jgguerre@uacam.mx http://orcid.org/0000-0002-2913-1309

José Duarte Méndez. Docente-investigador en la Universidad Vizcaya de las Américas (UVA), México, desde el 2016 hasta la actualidad. Se desempeña en asesoramiento de proyectos de investigación. Realiza investigación y gestión en temas acerca de servicios gerontogeriátricos.

Correo electrónico: josefranciscoduarte8@gmail.com

https://orcid.org/0000-0001-9102-8030 
Diana Moguel Ruz. Ingeniera Bioquímica con especialidad en alimentos. Cuenta con una maestría en Pedagogía. Desde hace 18 años labora en el Benemérito Instituto Campechano (IC) en la Escuela de Gastronomía como profesora de tiempo completo, asimismo pertenece al área de Investigación y Posgrado del IC.

Correo electrónico: diana.moguel@instcamp.edu.mx http://orcid.org/0000-0003-4088-6865

José Canto Ramírez. Licenciado en Psicología FES-Zaragoza, UNAM. Maestría en Pedagogía en la UPN- Ajusco. Doctor en Pedagogía por la UPN-Ajusco. Perfil PRODEP. SEP-UPN. México. Profesor de tiempo completo. Con 30 años de experiencia docente. Coautor de dos libros y de artículos.

Correo electrónico: cantoramirez@yahoo.com.mx http://orcid.org/0000-0002-8478-963X

Martín Bretón de la Loza. Doctorado en proyectos. Maestría en nutrición y dietética. Licenciatura en medicina veterinaria y zootecnia. Licenciatura en nutrición. Director administrativo del Centro de Evaluación Nutricional y de salud. Certificación Project management for results (PM4R) Federación internacional de educación física. Publicación de artículos.

Correo electrónico: martin.breton@uaem.mx http://orcid.org/0000-0001-9921-4014 